\title{
Sleep-disordered breathing in pregnancy
}

\author{
Rosalia Silvestri • Irene Aricò
}

Published online: 2 April 2013

(C) Springer Science+Business Media New York 2013

\begin{abstract}
Sleep-disordered breathing (SDB) during pregnancy has recently gained medical interest due to its consequences on maternal health and fetal outcome. In fact, although epidemiologic data are still scanty, several reports indicate that snoring and obstructive sleep apnea (OSA) during pregnancy are commonly associated with maternal hypertension, morbidly increased body mass index (BMI), diabetes mellitus and, at child birth, are correlated with an increased percentage of C-sections, preterm births with infants small for gestational age (SGA). OSA is an eminently treatable condition by means of assisted nocturnal ventilation with continuous positive air pressure (CPAP) devices. Diagnosis and treatment of this potential pregnancy complication should be thus more aggressively pursued in order to avoid harmful consequences to both mother and child.
\end{abstract}

Keywords Sleep-disordered breathing (SDB) · Pregnancy · Preeclampsia $\cdot$ Hypertension $\cdot \mathrm{C}$-section $\cdot$ Small for gestational age (SGA) $\cdot$ Low birth weight (LBW) $\cdot$ Preterm birth $\cdot$ Diabetes mellitus $\cdot$ Overweight $\cdot$ Berlin questionnaire $\cdot$ Snoring $\cdot$ Obstructive sleep apnea (OSA) · Central sleep apnea (CSA) · Estrogen · Progesterone · Continuous positive airway pressure (CPAP) · Epworth sleepiness scale $(\mathrm{ESS}) \cdot$ Intrauterine growth retardation (IGR) · Nocturnal polysomnography (NPSG)

\section{Introduction}

Sleep-disordered breathing (SDB) refers to abnormal breathing during sleep ranging from simple snoring to complete cessation of breathing, i.e. apnea, defined as an interruption

\section{R. Silvestri $(\triangle) \cdot \mathrm{I}$. Aricò}

Sleep Medicine Center, Department of Neurosciences, Messina University, AOU G. Martino, Via Consolare Valeria, 98124 Messina, Italy

e-mail: rosalia.silvestri@unime.it

I. Aricò

e-mail: i.arico@me.com of airflow for at least ten seconds, usually accompanied with oxygen desaturation and fragmented sleep [1], whereas a hypopnea requires a $30 \%$ reduction of airflow with associated 3-4 \% oxygen desaturation.

Arousal from sleep is indeed the mechanism that allows the resumption of normal breathing. Unfortunately the same mechanism is responsible for a sympathetic overdrive during sleep, leading progressively to chronic daytime hypertension. Other consequences of SDB include cardiac arrhythmias with a risk of atrial fibrillation, increased platelets aggregation and metabolic consequences including the metabolic syndrome [2]. Behavioral/emotional symptoms include fatigue, excessive daytime sleepiness (EDS), irritability and depressive mood. Sleep apnea syndrome is defined by an apnea-hypopnea index (AHI) of $>5$ per hour of sleep, linked to behavioral consequences. In obstructive sleep apnea (OSA) there is a maintained effort from the diaphragm and the accessory respiratory muscles to overcome the cessation of flow. Metabolic and cardiovascular consequences of OSA are detrimental to health and generally worse than those of central sleep apnea (CSA) where airflow interruption is correlated with a complete cessation of respiratory effort. The prevalence and severity of SDB is lower in women than in men. The estimated prevalence of OSA among women of reproductive age is around 5-6\% [3]. OSA prevalence is increased during pregnancy, especially in the third trimester, but the extent of this increase is still unknown since no epidemiological studies are available thus far to reveal it. Snoring has been reported to occur in $14-45 \%$ of pregnant women $[4,5]$. Symptoms and signs suggestive of SDB during pregnancy include, besides snoring and chocking during sleep, the typical behavioral symptoms described above. However, some of the latter may be compounded by typical symptoms of pregnancy including fatigue, fragmented sleep and reduced daytime functioning.

In this review we aim to discuss the reasons underlying an increased prevalence of SDB during pregnancy, a convenient way to diagnose it, treatment options and, above all, the potential detrimental consequences on maternal health and fetal outcome. 


\section{Physiopathogenetic mechanism of SDB in pregnant women}

Several factors contribute to a lower prevalence of SDB in women in their reproductive phase compared to men. They range from peculiar anatomic features including a small neck, peripheral rather than central distribution of fat, and hormonal effects on ventilatory control. Testosterone, in fact, is one of the main factors influencing AHI in men. Exogenous administration of testosterone increases AHI in men and is capable of inducing OSA in women [6].

As for the effects of female hormones on ventilation, estrogens hold no direct effect but play a permissive role for progesterone effects by increasing progesterone receptors, whereas progesterone stimulates ventilation during the luteal phase of the menstrual cycle, pregnancy or after exogenous administration via possible "resetting" of central chemoreceptors. Women with OSA have lower levels of 17hydroxyprogesterone [7]. Also, cardiovascular effects of apneas are different in women. Men are more susceptible than women to OSA-associated hypertension $[8,9]$. Hypertensive peaks after apneas in women are affected by the phase of the menstrual cycle. Several factors in pregnancy may contribute to an increased propensity for SDB. Among the latter are progressive weight gain and upward displacement of the diaphragm. Moreover, increased levels of estrogen may favor hyperemia, upper airway congestion via mucosal edema and vasomotor rhinitis. This would ultimately lead, in the course of the third trimester, to the narrowing of the airways thus increasing resistance to airflow [10]. Counteracting these anatomical negative factors, progesterone may play a protective role against SDB by promoting respiratory drive and minute ventilation. However this may ultimately promote respiratory alkalosis, which predisposes to the development of central apneas. The latter may also be caused by the instability of sleep that is typical of late pregnancy, with increased stage I and sleep phase transitions. Increased diaphragmatic effort as a consequence of a high respiratory drive would also cause suction pressure at the level of the upper airways, thus increasing collapsibility [11].

Functional residual capacity is also diminished by $20 \%$ as a consequence of the upper displacement of the diaphragm, resulting in reduced maternal oxygenation. This would in turn aggravate the altered alveolar-arterial oxygen gradient typical of late pregnancy [12]. Indeed, maternal oxygen reserves are scanty by the end of pregnancy due to a ventilation-perfusion mismatch, given the small airway closure at volumes greater than functional residual capacity.

Three major protective factors counteract the before mentioned negative ones: maternal postural changes to lateral position, reduced REM sleep [13] which usually enhances apneas, and the rightward shift of the oxy-hemoglobin dissociation curve allowing placental oxygen delivery despite the low maternal reserves.

\section{Epidemiology and risk factors of SDB in pregnant women}

OSA in pregnancy was first reported in 1978 [14]. Since then several case records have been published and even larger studies, mostly prospective $[15,16]$, or crosssectional $[17,18 \bullet \cdot$, were limited either by small samples or by lack of instrumental confirmation of apneas [19] prevalently based on self-reported symptoms derived from questionnaires.

SDB in women is often underdiagnosed on the basis of clinical symptoms, even when of moderate to grave severity, as demonstrated by the Wisconsin Sleep Cohort Study (WSCS, 2). Snoring is often underreported in women [20]. Female patients with OSA tend to emphasize fatigue, tiredness and lack of energy [21] rather than EDS [22]. Women with OSA show higher anxiety and depression scores than men [23]. On the other hand, OSA-related insomnia, chronic fatigue or depression may not be easily attributable to OSA, especially since women are less likely to be accompanied to the doctor's office by a bed partner whose complimentary sleep history is often important in identifying sleep symptoms.

SDB and obesity share a bidirectional relationship [24]: weight gain leads to upper airways narrowing, whereas SDB via EDS and daytime inactivity maintain and further promote weight gain. Also SDB-induced poor sleep quality correlate with leptin and insulin resistance, thus favoring obesity [25]. Weight gain and excessive fluid retention during pregnancy enhance the risk and prevalence of snoring from baseline through the third trimester, as shown in a recent prospective survey [26•].

\section{SDB diagnosis and screening tools}

All pregnant women at greater risk for SDB, including obese and hypertensive patients, should be systematically screened for OSA [27••]. The Berlin Questionnaire and the Epworth Sleepiness Scale (ESS) are commonly used to screen for OSA in nonpregnant populations. However, their value has been questioned in pregnancy [28•]. Nocturnal polisomnography (NPSG) is the ultimate tool to confirm and count apneas during sleep. It is also questionable which AHI should be considered abnormal during pregnancy. Should it be the usual cut off value of $\geq 5$ or else, given the limited time of pregnancy, 40 weeks, only a higher AHI 
would be able to substantially impact maternal and fetal wellbeing [27••]. Most recently, the same authors [29•] described a four variable model including frequent snoring, chronic hypertension, age and BMI, which performed significantly better than the classic association of Berlin Questionnaire and ESS, as proven by an ambulatory evaluation with the watch-PAT 100 (WP100), a wrist-mounted device to assess OSA diagnosis [30]. Other authors [27••] have conveniently used Index 1 of the multivariable apnea prediction index (MAPI, [31]) to screen for SDB in the last trimester of pregnancy.

One problem to solve is which women should undergo the ultimate screening tool, i.e. NPSG. In fact, on one side, Pien et al. [16] maintained that in the absence of clinical OSA symptoms, intrauterine growth retardation (IGR) gestational hypertension and uncomplicated snoring are insufficient clues to justify NPSG, whereas Santiago et al. [32] argue that in the absence of adequate epidemiologic studies on SDB in pregnancy, NPSG should be offered to all patients with hypertension and IGR associated to snoringrelated sleep alterations.

There is no evidence to suggest difficulties at performing NPSG in pregnant women and, in fact, several studies report of successful NPSG-based diagnoses in pregnant women $[33,34]$.

\section{Sleep-disordered breathing and adverse pregnancy outcomes}

SDB detrimental consequences include maternal and fetal adverse outcomes (see Table 1). Maternal consequences of SDB include fatigue, hypertension, diabetes mellitus, preeclampsia, increased percentage of C-sections.

SDB-induced maternal hypoxia may trigger oxidative stress and endothelial dysfunction, likely implicated in the pathogenesis of gestational hypertension [35]. Furthermore, the absence of the nocturnal physiological dipping of blood pressure is seen both in OSA and in preeclampsia, possibly being a mediator of the latter condition [36]. Endothelial dysfunction is also more likely to occur in pre-eclamptic women secondary to SDB [37]. Although several studies have shown increased frequency of preeclampsia in women

Table 1 Detrimental consequences of SDB in pregnancy

\begin{tabular}{ll}
\hline Maternal effects & Fetal consequences \\
\hline Maternal fatigue & Intrauterine growth retardation \\
Gestational hypertension & Small for gestational age \\
Gestational diabetes & Preterm birth \\
Preeclampsia & Hypoxic brain damage \\
C-section & \\
\hline
\end{tabular}

with snoring or flow limitations during pregnancy [18••, $27 \bullet \bullet, 38 \bullet \bullet$, it is still unclear whether upper airway changes precede the development of preeclampsia or else, preeclampsia diffuse edema secondary effect upper airways leading to inspiratory flow limitations [39]. The dimension of these effects are consistent with recent reports $[27 \bullet \bullet, 38 \bullet \bullet$ suggesting an adjuster OR from $1.60(95 \%$ CI, 2.16-11.26) to 2.3 (95\% CI, 1.4-4.0) for the likelihood of pregnancy-induced hypertension and preeclampsia associated with SDB.

A significant association of SDB with gestational diabetes after controlling for BMI at delivery, adjusted OR 2.1 (95\% CI, 1.3-3.4) [27•. ] suggests a different mechanism from obesity-dependent insulin resistance as indeed shown in a non-pregnant population sample where the dimension of SDB correlated with insulin resistance and glucose tolerance $[40,41]$.

There is an increased percentage of unplanned C-section delivery in pregnant women with SDB ranging from OR of 1.74 (95\% CI, 1.48-2.04) in the Taiwan population [38.•] to an OR of 2.1 (95\% CI, 1.4-3.2) in a European population-based study.

Even in this cases, the mechanism seemed to be independent from obesity. Interestingly, studies using actigraphy showed that sleep-deprived women at term had significantly longer labor and more C-sections [42], suggesting that sleep fragmentation itself rather than SDB could be related to an adverse outcome. Of notice, restless leg syndrome (RLS) or Willis Ekbom Disease (WED), a frequent sleep disorder during pregnancy, seems to be also related to the presence of snoring during pregnancy [43•], and may contribute to the before mentioned mechanism of sleep fragmentation thus potentiating SDB as well as C-section as a final delivery mode.

Fetal consequences of SDB have been well documented in experimental animals [44-46].

Maternal snoring during pregnancy is associated with enhanced fetal erythropoiesis $[47 \bullet \bullet]$ manifested by increased cord blood levels of circulating nRBC, IPO and IL-6. These subtle alterations in fetal wellbeing markers are independent from significant differences in birth weight or Apgar scores. It is plausible that SDB induced intermittent hypoxia is the intermediate mechanism, or else that the SDB secondary sympathetic overdrive effects placental perfusion leading to enhanced erythropoiesis.

Several studies suggest a link of SDB with preterm delivery $[27 \bullet \bullet, 47 \bullet \bullet]$ with an OR of $1.6(95 \% \mathrm{CI}, 1.1-3.2)$ and $2.3(95 \% \mathrm{CI}, 1.77-3.01)$ respectively.

Babies were also low birth weight (LBW) [19, 49] and small for gestational age (SGA, [47••]) with adjusted OR of 1.76 (95\% CI, 1.28-2.40) and 1.34 (95\% CI, 1.09-1.66), respectively. Previous studies reported lower Apgar scores at birth in pregnant women with OSA [48, 49]. 
The mechanisms explaining the link between OSA and adverse fetal outcome remain partially obscure. One possibility suggests that the frequency and severity of maternal SDB may be low enough to spare women from adverse effects, still be detrimental to the more oxygen-sensitive fetuses [19].

\section{Treatment of SDB during pregnancy}

Besides sleep hygiene recommendations (lateral positioning, restrained use of alcohol and sedatives) conservative treatment with CPAP may be successfully implemented and well tolerated during pregnancy after a NPSG-guided nocturnal titration. This would be an important goal (recommendation $\mathrm{C}$ ) to counteract maternal hypoxia. The use of CPAP in moderate to severe OSA ( $\mathrm{AHI} \geq 15)$ in the general population is commonly indicated (recommendation A).

Several authors have documented the positive effect of CPAP in pregnant women with SDB, even mild $(\mathrm{AHI} \geq 5)$, and on hypertension in the presence of clinical symptoms $[11,15,50,51]$ with improved cardiac output in preeclamptic women [52] and no evidence of adverse events.

However, a prospective longitudinal study evaluating early intervention of nasal CPAP therapy [51] demonstrated that this approach alleviated SDB symptoms in women but was not sufficient to prevent negative pregnancy outcomes. Women with OSA are often treated with suboptimal CPAP levels [53]. Also females treated with CPAP show, in general, higher mortality risk than men (OR 3.44) due to greater comorbidity [54].

The use of oral appliances appears difficult during pregnancy and is not to be pursued unless CPAP is not tolerated.

Furthermore, due to the risk of surgical procedures and anesthesia during pregnancy, upper airway surgery should be avoided.

Supplemental night oxygen is not supported by evidence and could potentially be detrimental by prolonging apnea duration.

\section{Conclusions}

SDB during pregnancy confers several risks to the maternal and fetal wellbeing through different mechanisms associated with intermittent hypoxia, sleep alterations, metabolic and hemodynamic changes. It is therefore mandatory to diagnose this condition in the presence of potential risk factors or of suggestive symptoms, in order to implement therapy and avoid possible harm to the mother and the developing fetus.
Conflict of Interest Rosalia Silvestri declares no conflict of interest. Irene Aricò declares no conflict of interest.

\section{References}

Papers of particular interest, published recently, have been highlighted as:

- Of importance

-• Of major importance

1. Iber C A-IS. Chesson A. Quan SF. The AASM manual for the scoring of sleep and associated events: rules, terminology and technical specifications. 1st edition. Westchester (IL): American Academy of Sleep Medicine. 2007.

2. Vgontzas AN, Bixler EO, Chrousos GP. Sleep apnea is a manifestation of the metabolic syndrome. Sleep Med Rev. 2005;9(3):211-24.

3. Young T, Palta M, Dempsey J, et al. The occurrence of sleepdisordered breathing among middle-aged adults. N Eng J Med. 1993;328:1230-5.

4. Calaora-Tournadre D, Ragot S, Meurice JC, et al. Obstructive sleep apnea syndrome during pregnancy: prevalence of main symptoms and relationship with pregnancy induced-hypertension and intrauterine growth retardation. Rev Med Interne. 2006;27:291-5.

5. Shuttle S, Del Conte A, Gross A, et al. Self-reported snoring and sleep in high risk pregnancies. Sleep Res. 1993;24:342.

6. Dexter DD, Dovre EJ. Obstructive sleep apnea due to endogenous testosterone production in a woman. Mayo Clin Proc. 1998;73(3):246-8.

7. Martins AB, Tufik S, Moura SM. Physiopathology of obstructive sleep apnea-hypopnea syndrome. J Bras Pneumol. 2007;33(1):93-100.

8. Hedner J, Bengtsson-Boström K, Peker Y, et al. Hypertension prevalence in obstructive sleep apnoea and sex: a populationbased case-control study. Eur Respir J. 2006;27:564-70.

9. Mohsenin V, Yaggi HK, Shah N, et al. The effect of gender on the prevalence of hypertension in obstructive sleep apnea. Sleep Med. 2009;10(7):759-62.

10. Izci B, Vennelle M, Liston WA, et al. Sleep-disordered breathing and upper airway size in pregnancy and post-partum. Eur Respir J. 2006;27(2):321-7.

11. Edwards N, Middleton PG, Blyton DM, et al. Sleep disordered breathing and pregnancy. Thorax. 2002;57:555-8.

12. Awe RJ, Nicotra MB, Newsom TD, et al. Arterial oxygenation and alveolar-arterial gradients in term pregnancy. Obstet Gynecol. 1979;53:182-6.

13. Brunner DP, Munch M, Biedermann K, et al. Changes in sleep and sleep electroencephalogram during pregnancy. Sleep. 1994; 17:576-82.

14. Joel-Cohen SJ, Schoenfield A. Fetal responses to periodic sleep apnea: a new syndrome in obstetrics. Eur J Obste Gynecol Reprod Biol. 1978;8:77-81.

15. Guilleminault C, Kreutzer M, Chang JL. Pregnancy, sleep disordered breathing and treatment with nasal continuous positive airway pressure. Sleep Med. 2004;5:43-53.

16. Pien GW, Fife D, Pack AI, et al. Changes in symptoms of sleep disordered breathing during pregnancy. Sleep. 2005;28:1299-305.

17. Izci B, Martin SE, Dundas KC, et al. Sleep complaints, snoring and daytime sleepiness in pregnant and pre-eclamptic women. Sleep Med. 2005;6:163-9.

18. •• Bourjelly G, Raker CA, Chalhoub M, et al. Pregnancy and fetal outcomes of symptoms of sleep-disordered breathing. Eur Respir J. 
2010;36:849-55. This is a wide cross-sectional survey that retrospectively examines post-partum females for the likelihood of pregnancy and fetal adverse events in relation to maternal SDB.

19. Venkata C, Venkateshiah SB. Sleep-disordered breathing during pregnancy. JABFM. 2009;22:158-68.

20. Redline S, Kump K, Tishler PV, et al. Gender differences in sleep disordered breathing in a community-based sample. Am J Respir Crit Care Med. 1994;149:722-6.

21. Chervin RD. Sleepiness, fatigue, tiredness, and lack of energy in obstructive sleep apnea. Chest. 2000;118:372-9.

22. Gottlieb DJ, Whitney CW, Bonekat WH, et al. Relation of sleepiness to respiratory disturbance index: the Sleep Heart Health Study. Am J Respir Crit Care Med. 1999;159:502-7.

23. Valipour A, Lothaller H, Rauscher H, et al. Gender-related differences in symptoms of patients with suspected breathing disorders in sleep: a clinical population study using the sleep disorders questionnaire. Sleep. 2007;30:312-9.

24. Shah N, Roux F. The relationship of obesity and obstructive sleep apnea. Clin Chest Med. 2009;30:455-65. vii.

25. Wolk R, Shamsuzzaman AS, Somers VK. Obesity, sleep apnea, and hypertension. Hypertension. 2003;42:1067-74.

26. - Facco FL, Grobman WA, Kramer J, et al. Self-reported short sleep duration and frequent snoring in pregnancy: impact on glucose metabolism. Am J Obstet Gynecol. 2010;203:142. This paper provides new insight on potential mechanisms for gestational diabetes in pregnant women with SDB.

27. • Facco FL. Sleep-disordered breathing in pregnancy. Semin Perinatol. 2011;35:335-9. This paper provides an up-to-date review of SDB and pregnancy with special emphasis on diagnosis and treatment.

28. - Olivarez SA, Maheshwari B, McCarthy M, et al. Prospective trial on obstructive sleep apnea in pregnancy and fetal heart rate monitoring. Am J Obstet Gynecol. 2010;202:e551-7. This originally designed study demonstrates fetal arrhythmias depending on maternal $S D B$.

29. - Facco FL, Ouyang DW, Zee PC, et al. Development of a pregnancy-specific screening tool for sleep apnea. J Clin Sleep Med. 2012;8:389-94. This is an original validation study proposing a novel screening tool for the diagnosis of OSA in pregnant women.

30. Kang D, Ye JY, Zheng L, et al. Evaluation of Watch PAT as a diagnosing test for patients with obstructive sleep apnea hypopnea syndrome. Zhonghua Er Bi Yan Hou Tou Jing Wai Ke Za Zhi. 2012;47:813-6.

31. Maislin G, Pack AI, Kribbs NB, et al. A survey screen for prediction of apnea. Sleep. 1995;18:158-66.

32. Santiago JR, Nolledo MS, Kinzler W, et al. Sleep and sleep disorders in pregnancy. Ann Intern Med. 2001;134:396-408.

33. Edwards N, Blyton DM, Kirjavainen TT, et al. Hemodynamic responses to obstructive respiratory events during sleep and augmented in women with preeclampsia. Am J Hypertens. 2001;14:1090-5.

34. Poyares D, Guilleminault C, Hachul H, et al. Preeclampsia and nasal CPAP: part 2. Hypertension during pregnancy, chronic snoring, and early nasal CPAP intervention. Sleep Med. 2007;9:15-21.

35. Dekker GA, Sibai BM. Etiology and pathogenesis of preeclampsia: current concepts. Am J Obstet Gynecol. 1998;179:1359-75.

36. Beilin LJ, Deacon J, Michael CA, et al. Diurnal rhythms of blood pressure, plasma renin activity, angiotensin II and catecholamines in normotensive and hypertensive pregnancies. Clin Exp Hypertens B. 1983;2:271-93.
37. Yinon D, Lowenstein L, Suraya S, et al. Preeclampsia is associated with sleep-disordered breathing and endothelial dysfunction. Eur Respir J. 2006;27:328-33.

38. • Chen YH, Kang JH, Lin CC, et al. Obstructive sleep apnea and the risk of adverse pregnancy outcomes. Am J Obstet Gynecol. 2012;206:136. This is an important epidemiologic paper regarding eastern populations with different risk factors and outcomes compared to the western counterpart.

39. Kapsimalis F, Kryger M. Obstructive sleep apnea in pregnancy. Sleep Med Clin. 2007;2:603-13.

40. Punjabi NM, Shahar E, Redline S, et al. Sleep-disordered breathing, glucose intolerance, and insulin resistance: the Sleep Hearth Health Study. Am J Epidemiol. 2004;160:521-30.

41. Okada M, Takamizawa A, Tsushima K, et al. Relationship between sleep-disordered breathing and lifestyle-related illnesses in subjects who have undergone health-screening. Intern Med. 2006; 45:891-6.

42. Lee KA, Gay CL. Sleep in late pregnancy predicts length of labor and type of delivery. Am J Obstet Gynecol. 2004;191:2041-6.

43. - Sarberg M, Josefsson A, Wirehn AB, et al. Restless legs syndrome during and after pregnancy and its relation to snoring. Acta Obstet Gynecol Scand. 2012;91:850-5. This article stresses the importance of $S D B$ comorbidity with other sleep disorders and the additive contribution to sleep fragmentation.

44. Gozal D, Gozal E, Reeves SR, et al. Gasping and autoresuscitation in the developing rat: effect of antecedent intermittent hypoxia. J Appl Physiol. 2002;92:1141-4.

45. Schwartz JE, Kovach A, Meyer J, et al. Brief, intermittent hypoxia restricts fetal growth in Sprague-Dawley rats. Biol Neonate. 1998;73:313-9.

46. Gozal D, Reeves SR, Row BW, et al. Respiratory effects of gestational intermittent hypoxia in the developing rat. Am J Respir Crit Care Med. 2003;167:1540-7.

47. •- Tauman R, Many A, Deutsch V, et al. Maternal snoring during pregnancy is associated with enhanced fetal erythropoiesis-a preliminary study. Sleep Med. 2011;12:518-22. This article focuses on subtle changes in markers of fetal wellbeing demonstrating their alteration prior to any clinical adverse outcome.

48. Champagne KA, Kimoff RJ, Barriga PC, et al. Sleep disordered breathing in women of childbearing age and during pregnancy. Indian J Gynaecol Obstet. 2008;100:141-6.

49. Franklin KA, Holmgren PA, Jonsson F, et al. Snoring, pregnancyinduced hypertension, and growth retardation of the fetus. Chest. 2000;117:137-41.

50. Poyares D, Guilleminault C, Hachul H, et al. Pre-eclampsia and nasal CPAP: Part 2. Hypertension during pregnancy, chronic snoring, and early nasal CPAP intervention. Sleep Med. 2007;9:15-21.

51. Guilleminault C, Palombini L, Poyares D, et al. Pre-eclampia and nasal CPSP: Part 1. Early intervention with nasal CPAP in pregnant women with risk-factors for pre-eclampsia: preliminary findings. Sleep Med. 2007;9:9-14.

52. Blyton DM, Sullivan CE, Edwards N. Reduced nocturnal cardiac output associated with preeclampsia is minimized with the use of nocturnal nasal CPAP. Sleep. 2004;27:79-84.

53. Yukawa K, Inoue Y, Yagyu H, et al. Gender differences in the clinical characteristics among Japanese patients with obstructive sleep apnea syndrome. Chest. 2009;135:337-43.

54. Morrish E, Shneerson JM, Smith IE. Why does gender influence survival in obstructive sleep apnoea? Respir Med. 2008;102: $1231-6$. 